\title{
Convective heat and solute transfer in Newtonian fluid saturated inclined porous cavity
}

\author{
Latreche Abdelkrim *, Djezzar Mahfoud \\ Département de Physique, Université Mentouri Constantine1 \\ *Corresponding author E-mail: abdelkrim.latreche@gmail.com
}

Copyright $\odot 2014$ Latreche Abdelkrim, Djezzar Mahfoud. This is an open access article distributed under the Creative Commons Attribution License, which permits unrestricted use, distribution, and reproduction in any medium, provided the original work is properly cited.

\begin{abstract}
This paper summarizes a numerical study of double-diffusive natural convection in square inclined cavity filled with fluid saturated porous media. Transverse gradients of heat and solute are applied on the two horizontal walls of the cavity, while the other two walls are impermeable and adiabatic. The Darcy model with the Boussinesq approximation is used to solve the governing equations. The flow is driven by a combined buoyancy effect due to both temperature and concentration variations. A finite volume approach has been used to solve the non-dimensional governing equations. The results are presented in streamline, isothermal, iso-concentration, Nusselt and Sherwood contours for different values of the non-dimensional governing parameters.
\end{abstract}

Keywords: Boussinesq Approximation, Darcy Model, Finite Volumes, Inclined Cavity, Natural Convection, Porous Media.

\section{Introduction}

The study of double-diffusive natural convection in fluid-saturated porous media has been motivated by its wide range of applications in many engineering fields such as the migration of moisture through air contained in fibrous insulations and the underground spreading of chemical contaminants through water-saturated soil. A comprehensive review of the natural convection due to combined thermal and solutal driving forces was conducted by Nield and Bejan [1], Ingham and Pop [2]. Most of the existing studies in the literature on double diffusive convection are concerned with rectangular cavities where the temperature and concentration gradients are either vertical or horizontal. The mass transfer resulting from high convection in a porous medium heated from below has been studied analytically and numerically by Trevisan and Bejan [3], they indicate the existence of different scaling laws for the dependence of the Nusselt number versus the Rayleigh and Lewis numbers. Mamou and Vasseur [4] studied numerically and theoretically the thermo-solutal bifurcation phenomena in porous enclosures subject to vertical temperature and concentration gradients. Numerical results of thermo-solutal natural convection have been reported by Bourich et al. [5] in the case of a horizontal porous cavity partially heated from below and differentially salted. It was found that multiple solutions are possible in pure thermal convection vanishes in the presence of horizontal solutal gradients when critical conditions, depending on the Rayleigh and Lewis number, are reached. Double diffusive convection in a porous enclosure submitted to cross gradients of temperature and concentration has been studied by the same author [6], the effects of the governing parameters on the flow structure and heat and mass transfer are analyzed. It is demonstrated that the solutal buoyancy force induced by horizontal concentration gradients eliminates the multiplicity of solutions obtained in pure thermal convection when $\mathrm{N}$ exceeds some critical value, which depends on Le and Ra.

Mohamad and Bennacer [7] studied numerically the existence of multiple solutions in a horizontal porous enclosure heated horizontally and salted from the bottom. It was demonstrated that the multiplicity of solution obtained when $\mathrm{Gr}_{\mathrm{m}}$ (modified Grashof number) $=1000$ and $\mathrm{N}$ varying in the range $0.8 \leq \mathrm{N} \leq 1$. It was observed that the bifurcation from monocellular dominating flow to bicellular dominating flow in this range of $\mathrm{N}$, and in the case of thermally driven flow the concentration gradient reversal was possible. The onset of double diffusive convection in a horizontal porous cavity is studied numerically by Mahidjiba et al. [8] using linear stability analysis. Mixed boundary conditions for heat and solute are specified on the horizontal walls of the enclosure while the two vertical ones are impermeable and adiabatic. 
It is shown that there exists a supercritical Rayleigh number for the onset of the supercritical convection and an over stable Rayleigh number, at which over stability may arise. The over stable regime is shown to exist up to a critical Rayleigh number at which the transition from the oscillatory to direct mode convection occurs. The existence of multiple steady-state solutions, for a given set of the governing parameters, was demonstrated by Kalla et al. [9] in a numerical and analytical study of double-diffusive natural convection within a horizontal porous layer, where the vertical and the horizontal walls are submitted respectively to uniform heat and mass fluxes. Costa [10] studied numerically the double-diffusive natural convection problem in parallelogrammic enclosures filled with fluid-saturated porous media. Vertical walls are maintained at constant deferent levels of temperature and concentration, and the inclined walls are adiabatic and impermeable. It is shown that in terms of flow structure, temperature levels and concentration levels, strong changes occur in the parallelogrammic enclosure when changes are made on the Darcymodified Rayleigh number, on the inclination angle and on the aspect ratio of the enclosure. Very different behaviors are obtained for the combined or opposite global heat and mass flows that cross the parallelogrammic enclosure.

A literature review shows that relatively little work is available on the case of natural convection in inclined enclosures. Therefore, the present paper investigates numerically double diffusive natural convection within a porous inclined cavity with localized heating and salting from below. The complete system of governing equations is solved numerically and results are obtained for a large range of the governing parameters. The global Nusselt and Sherwood numbers dependence on the dimensionless governing parameters and boundary conditions is explored in detail.

\section{Mathematical formulation}

The studied configuration, depicted in Fig.1, is a square-saturated porous cavity with length H. The cavity is tilted at an angle $\alpha$ with respect to the horizontal plane. The wall at $\mathrm{Y}=\mathrm{H}$ represents the low-temperature $\left(\mathrm{T}_{1}\right)$ and low-concentration $\left(\mathrm{S}_{1}\right)$ boundary, and the wall at $\mathrm{Y}=0$ denotes the high temperature $\left(\mathrm{T}_{\mathrm{h}}\right)$ and high concentration $\left(\mathrm{S}_{\mathrm{h}}\right)$ boundary. The other two walls are regarded as being insulated and impermeable. It is assumed that the third dimension of the cavity is large enough so that the fluid flow and heat and mass transfer can be considered two-dimensional. Hypotheses of incompressible and laminar flow are considered, and the saturated porous medium is assumed isotropic and homogeneous with constant thermo physical properties. Interaction between the thermal and concentration gradients, (Soret and Dufour effects) are neglected. The binary fluid that saturates the porous matrix is modeled as a Boussinesq incompressible fluid whose density variation can be expressed as:

$$
\begin{aligned}
& \rho=\rho_{0}\left[1-\beta_{T}\left(T-T_{0}\right)-\beta_{S}\left(S-S_{0}\right)\right] \\
& \beta_{T}=-\frac{1}{\rho_{0}}\left(\frac{\partial \rho}{\partial T}\right)_{P, S},
\end{aligned}
$$

The following non dimensional variables are introduced:

$$
x^{+}=\frac{x}{H}, \quad y^{+}=\frac{y}{H}, \quad u^{+}=\frac{u}{a / H}, \quad v^{+}=\frac{v}{a / H}, \quad \psi^{+}=\frac{\psi}{a}, \quad T^{+}=\frac{T-T_{l}}{T_{h}-T_{l}}, \quad S^{+}=\frac{S-S_{l}}{S_{h}-S_{l}}
$$

The dimensionless governing equations, based on the above definitions, are as follows:

$$
\frac{\partial \mathrm{u}^{+}}{\partial \mathrm{x}^{+}}+\frac{\partial \mathrm{v}^{+}}{\partial \mathrm{y}^{+}}=0
$$

$\left(\frac{\partial^{2}}{\partial \mathrm{x}^{+2}}+\frac{\partial^{2}}{\partial \mathrm{y}^{+2}}\right) \psi^{+}=-\mathrm{Ra}^{*} *\left(\cos \alpha\left(\frac{\partial \mathrm{T}^{+}}{\partial \mathrm{x}^{+}}+\mathrm{N} \frac{\partial \mathrm{S}^{+}}{\partial \mathrm{x}^{+}}\right)-\sin \alpha\left(\frac{\partial \mathrm{T}^{+}}{\partial \mathrm{y}^{+}}+\mathrm{N} \frac{\partial \mathrm{S}^{+}}{\partial \mathrm{y}^{+}}\right)\right)$

$\mathrm{u}^{+} \frac{\partial \mathrm{T}^{+}}{\partial \mathrm{x}^{+}}+\mathrm{v}^{+} \frac{\partial \mathrm{T}^{+}}{\partial \mathrm{y}^{+}}=\left(\frac{\partial^{2} \mathrm{~T}^{+}}{\partial \mathrm{x}^{+2}}+\frac{\partial^{2} \mathrm{~T}^{+}}{\partial \mathrm{y}^{+2}}\right)$

$\mathrm{u}^{+} \frac{\partial \mathrm{S}^{+}}{\partial \mathrm{x}^{+}}+\mathrm{v}^{+} \frac{\partial \mathrm{S}^{+}}{\partial \mathrm{y}^{+}}=\frac{1}{\mathrm{Le}}\left(\frac{\partial^{2} \mathrm{~S}^{+}}{\partial \mathrm{x}^{+2}}+\frac{\partial^{2} \mathrm{~S}^{+}}{\partial \mathrm{y}^{+2}}\right)$

The dimensionless boundary conditions are:

$\forall \mathrm{x}^{+}, \mathrm{y}^{+}=0: \mathrm{T}^{+}=1, \mathrm{~S}^{+}=1, \psi^{+}=0$

$\forall \mathrm{x}^{+}, \mathrm{y}^{+}=1: \mathrm{T}^{+}=0, \mathrm{~S}^{+}=0, \psi^{+}=0$

$\forall \mathrm{y}^{+}, \mathrm{x}^{+}=0$ and $1: \frac{\partial \mathrm{T}^{+}}{\partial \mathrm{x}^{+}}=\frac{\partial \mathrm{S}^{+}}{\partial \mathrm{x}^{+}}=\psi^{+}=0$

From the dimensionless equations it is seen that the present problem is governed by three dimensionless parameters: the buoyancy ratio $\mathrm{N}$, the Lewis number $\mathrm{Le}$ and the thermal Rayleigh number $\mathrm{Ra}_{\mathrm{T}}$ defined as: $R a_{T}=\frac{g H \beta_{T} K \Delta T}{a v}, \quad N=\frac{\beta_{S} \Delta S}{\beta_{T} \Delta T}, L e=\frac{a}{D}$ 


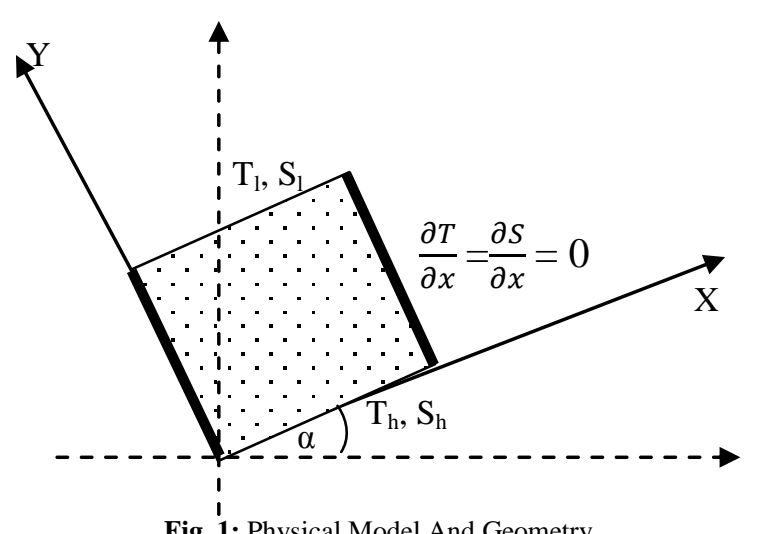

The average values of Nusselt and Sherwood numbers, evaluated on the bottom wall are given by:

$N u=-\left.\int_{0}^{1} \frac{\partial T^{+}}{\partial y^{+}}\right|_{Y^{+}=0} d x^{+} \quad, \quad S h=-\left.\int_{0}^{1} \frac{\partial S^{+}}{\partial y^{+}}\right|_{Y^{+}=0} d x^{+}$

\section{Numerical solution}

The numerical solution of governing equations (4)-(7) with specified boundary conditions equations (8) is obtained using the volume finite method described by Patankar [11]. The computation domain is divided into rectangular control volumes with one grid located at the centre of the control volume that forms a basic cell. The set of conservation equations are integrated over the control volumes, leading to a balance equation for the fluxes at the interface.

The iterative process, employed to find the stream function, temperature and concentration fields, was repeated until the following convergence criterion was satisfied

$$
\frac{\sum_{i} \sum_{j}\left(\Phi_{i, j}^{\text {new }}-\Phi_{i, j}^{\text {old }}\right)}{\sum_{i} \sum_{j} \Phi_{i, j}^{\text {new }}} \leq 10^{-6}
$$

Where $\Phi$ stand for $\Psi, \mathrm{T}$ and $\mathrm{S}$. The subscripts $\mathrm{i}$ and $\mathrm{j}$ denote grid locations in the (x, y) plane. A further decrease of the convergence criteria $10^{-6}$ does not cause any significant change in the final results. Numerical tests, using various mesh sizes, were done for the same conditions in order to determine the best compromise between accuracy of the results and computer time. A mesh size of $61 \times 61$ was adopted. The accuracy of the code was checked, modifying the thermal and solutal boundary conditions, to reproduce the results reported in [6]. Good agreement can be seen from Table 1 with a maximum deviation of about $3.4 \%$.

Table 1: Validation of the Numerical Code, for $\alpha=0, \mathrm{Ra}=200, \mathrm{~N}=0,3$ and Various Le, in Terms of $\psi$ Max, $\mathrm{Nu}$ and $\mathrm{Sh}$

\begin{tabular}{|c|c|c|c|c|c|c|}
\hline Le & \multicolumn{2}{|c|}{$\psi_{\max }$} & \multicolumn{2}{c|}{ Nu } & \multicolumn{2}{c|}{ Sh } \\
\hline & Present work & Ref.[6] & Present work & Ref.[6] & Present work & Ref.[6] \\
\hline 0.1 & 11.625 & 11.706 & 4.484 & 4.633 & 1.209 & 1.221 \\
\hline 1 & 9.505 & 9.609 & 4.130 & 4.276 & 4.840 & 5.086 \\
\hline 10 & 9.104 & 9.171 & 3.983 & 4.078 & 15.870 & 17.02 \\
\hline
\end{tabular}

\section{Results and discussion}

\subsection{Considered situations}

There are four parameters governing the problem under analysis: Le, $\mathrm{N}, \mathrm{Ra}, \alpha$. All the presented results refer to moist air saturating the porous medium, with a low concentration of water vapor, thus fixing $\mathrm{Le}=0.8$. Many values for $\mathrm{N}$, Ra and $\alpha$ in this work being taken $\mathrm{N}=(5,2,0.5$ and 0$), \mathrm{Ra}=\left(100\right.$ and 50) and $\alpha=\left(0^{\circ}, 30^{\circ}, 45^{\circ}, 60^{\circ}\right.$ and $\left.90^{\circ}\right)$.

\subsection{Flow structure, temperature and concentration fields, and heat and mass transfer visualization.}

An obvious characteristic of convection in the porous material is the appearance of single or multiple cell flows. The physical notion of a cell is associated with an identifiable body of fluid rotating in the same sense. Therefore, it has to be bounded by a closed streamline within which the vortices are of the same sign. Positive and negative streamlines $\Psi$ correspond to counter clockwise and clockwise circulations, respectively. The convective motion will be referred to as 
natural flow whenever the fluid is ascendant above the heated element. On the other hand the convective pattern will be called anti natural when the fluid is descendant above the heated element.

Depending on the tilt angle $(\alpha)$ and the Darcy-Rayleigh number $(\mathrm{Ra})$, single or multiple cell convection was found. For $\mathrm{N}=0$ (In the case of no solute transfer) and in the ranges $0^{\circ} \leq \alpha \leq 90^{\circ}$ and $\mathrm{Ra}<100$ the single cell mode was obtained. An example of this flow is given in Fig. 2 where the streamlines and isotherms are shown.

The stream function shows a single extremum value whose magnitude becomes larger as $\mathrm{Ra}$ increases, indicating a more vigorous motion, as expected. As a function of the tilt angle, the $\psi$ extremum value presents a maximum around $45^{\circ}$.

Results for $\alpha=30^{\circ}, \mathrm{Ra}=100$ and combined global heat and mass flows are presented in Fig. $3 \mathrm{a}$ for $\mathrm{N}=2$ and in Fig. $3 \mathrm{~b}$ for $\mathrm{N}=5$. Main changes from Fig. 2 to 3 are due to the increase on the buoyancy term. Flow is more intense, the temperature and concentration gradients are higher near the horizontal walls and heat and mass transfer increases as $\mathrm{N}$ increases. As $\mathrm{N}$ increases, heat flows in a narrow region close to the right wall of the enclosure. In what concerns temperature and concentration fields, as Le $=0,8 \sim 1$, there are no major differences on these fields.

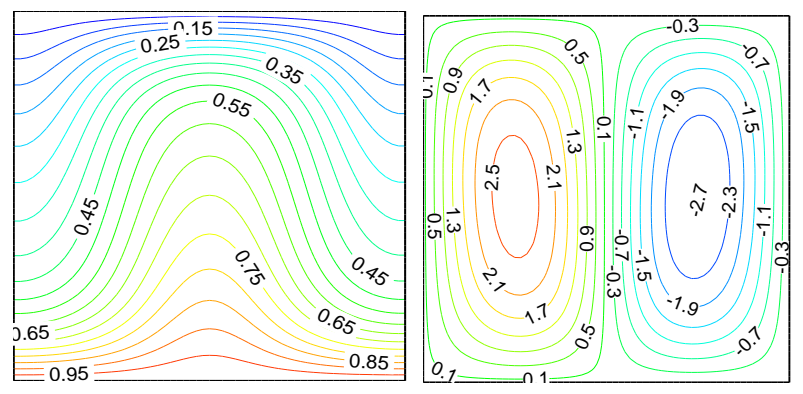

A
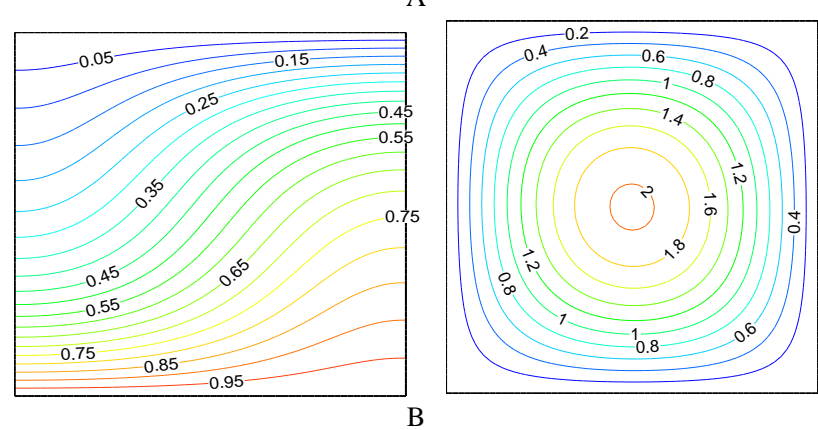

$\mathrm{B}$

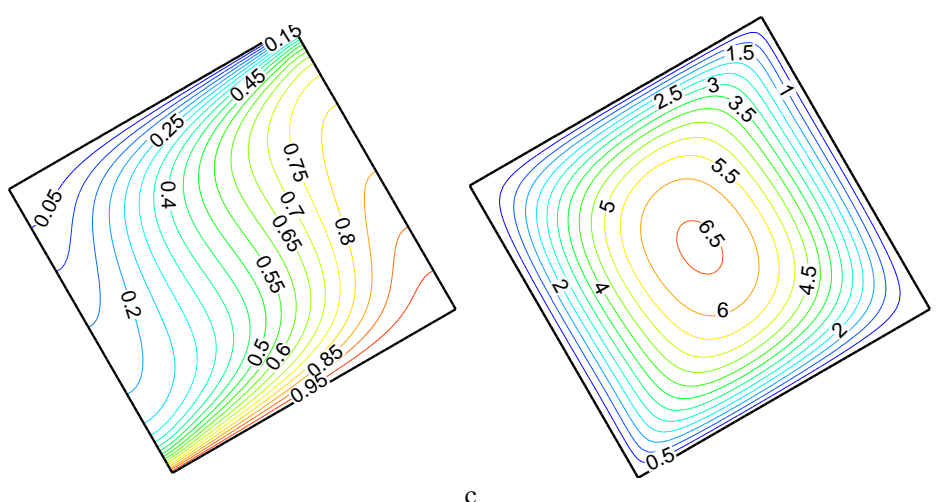

Fig. 2: Isotherms and Streamlines and For $\mathrm{N}=0$ (A) $\mathrm{Ra}=100, \alpha=0, \Psi \min =-2.702, \Psi \max =2.713$, (B) $\alpha=0, \mathrm{Ra}=50, \Psi \min =0, \Psi \max =2.037$, (C) $\alpha=30^{\circ}, \mathrm{Ra}=100, \Psi \min =0, \Psi \max =6.59$. 


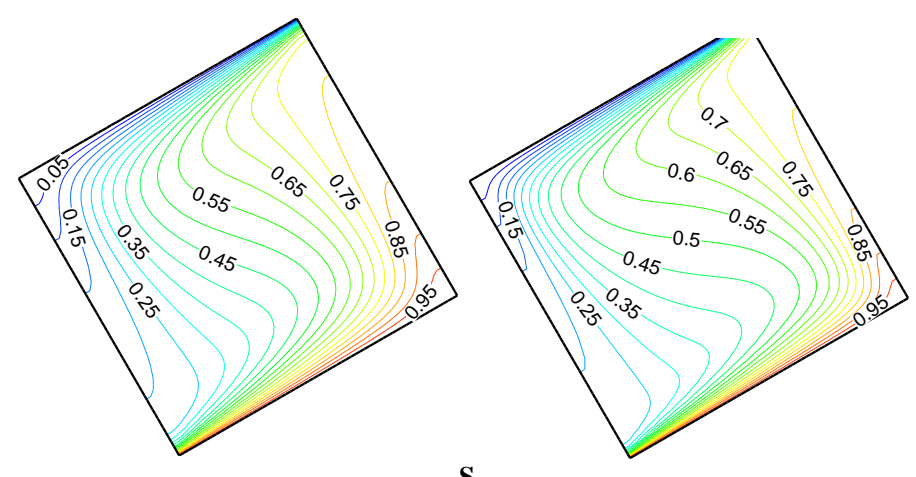

$\mathbf{S}$

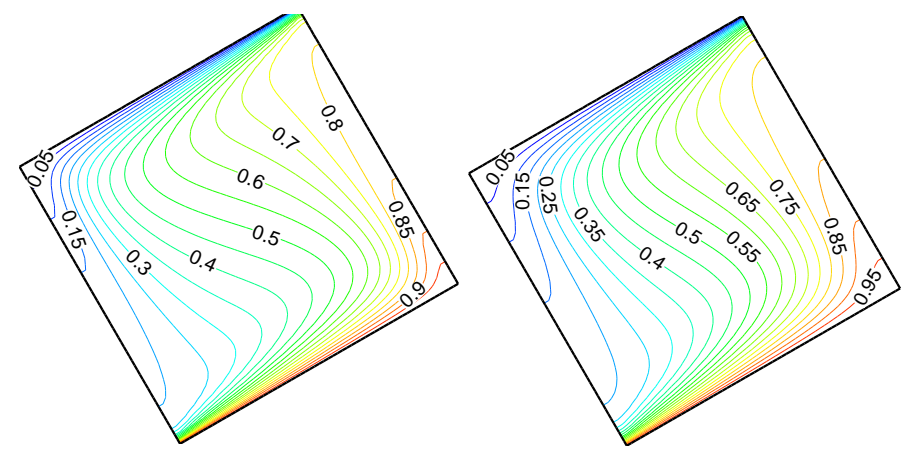

$\psi$

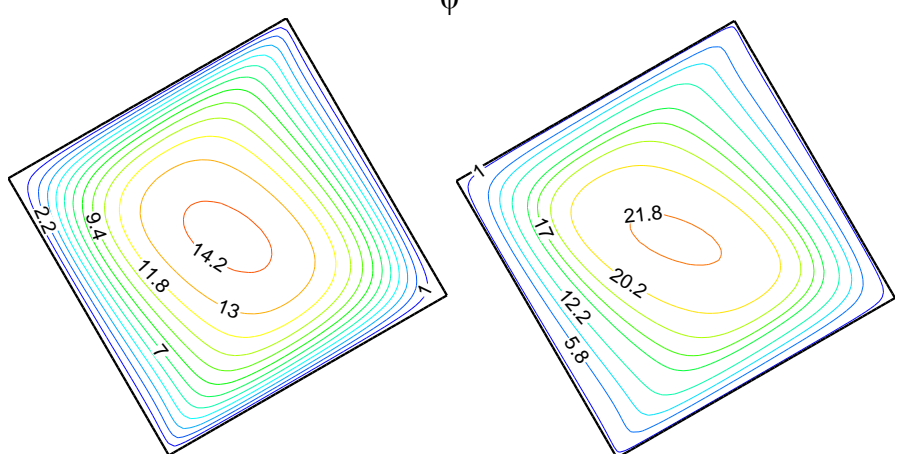

Fig. 3: Isotherms, Isoconcentration and Streamlines for Combined Global Heat and Mass Flows, $\mathrm{Le}=0,8, \alpha=30, \mathrm{Ra}=100$, for $(\mathrm{A}) \mathrm{N}=2$, $\Psi \mathrm{min}=0$, $\Psi \max =14.48$, (B) $\mathrm{N}=5, \Psi \min =0, \Psi \max =21.98$.

\subsection{Heat and mass transfer parameters}

Global Nusselt number for the situation of no solute transfer $(\mathrm{N}=0)$ is presented in Fig. 4, as function of the inclination angle $\alpha$ for Darcy-modified Rayleigh numbers of 50 and 100. The Nusselt number for $\mathrm{Ra}=100$ being always greater than for $\mathrm{Ra}=50$. For low values of $\alpha\left(\alpha \approx 0^{\circ}\right)$ and high values of $\alpha\left(\alpha \approx 90^{\circ}\right)$ the global Nusselt number is nearly the same. When $\left(0 \leq \alpha \leq 45^{\circ}\right)$ the Nusselt number increases for any value of $\mathrm{Ra}$. It is observed the existence of a maximum Nusselt number for $\alpha$ near $45^{\circ}$ and a minimum for $\alpha$ near $0^{\circ}$. A physical explanation can be given for the thermal diode effect. The hot fluid moves upwards and reaches the right wall, which has a favorable inclination, allowing some tangentiality to the flow flowing along the wall towards the cold wall. The same applies also for the descending cold fluid on the neighboring of the opposite horizontal wall. The flow is intense and the thermal gradients near the horizontal walls are high, thus resulting into high global heat transfer rates. 


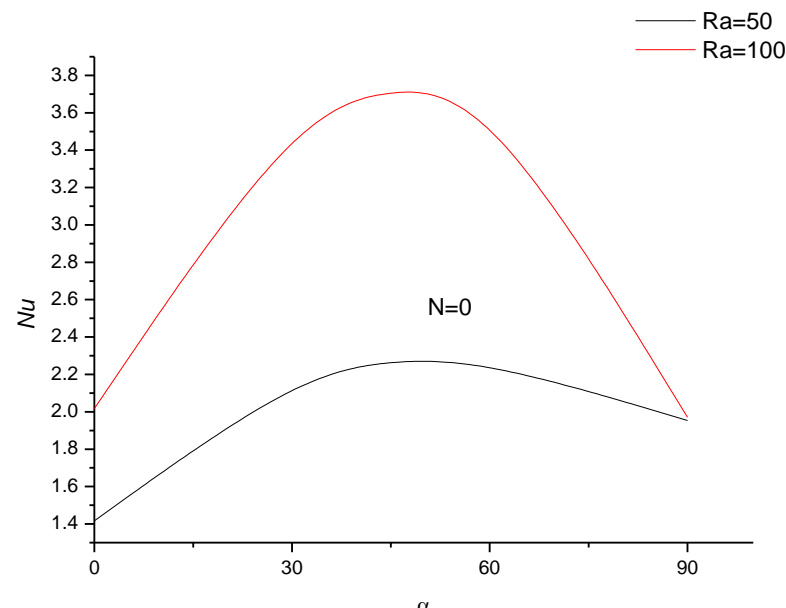

Fig. 4: Global Nusselt Number versus Inclination Angle $\alpha$ and For the Situation of Non-Solute Transfer $(\mathrm{N}=0)$ for $\mathrm{Ra}=50$ And 100.

Results for combined global heat and mass flows and $\mathrm{N}=0.5,2$ and 5 are presented in Fig. 5a-b and c respectively for the same values of Ra of 50 and 100 . In general terms, it can be observed that as Le $=0.8 \approx 1$, there are no significant differences between the behavior and the numeric values of the global Nusselt and Sherwood numbers. The highest heat and mass transfer parameters occurring for the range $40^{\circ} \leq \alpha \leq 60^{\circ}$ ) and the minimum Nusselt and Sherwood numbers correspond to $\alpha=0^{\circ}$. It is also observed that the increasing of the buoyancy ratio, always leads to increases on the heat and mass transfer performances of the enclosure.
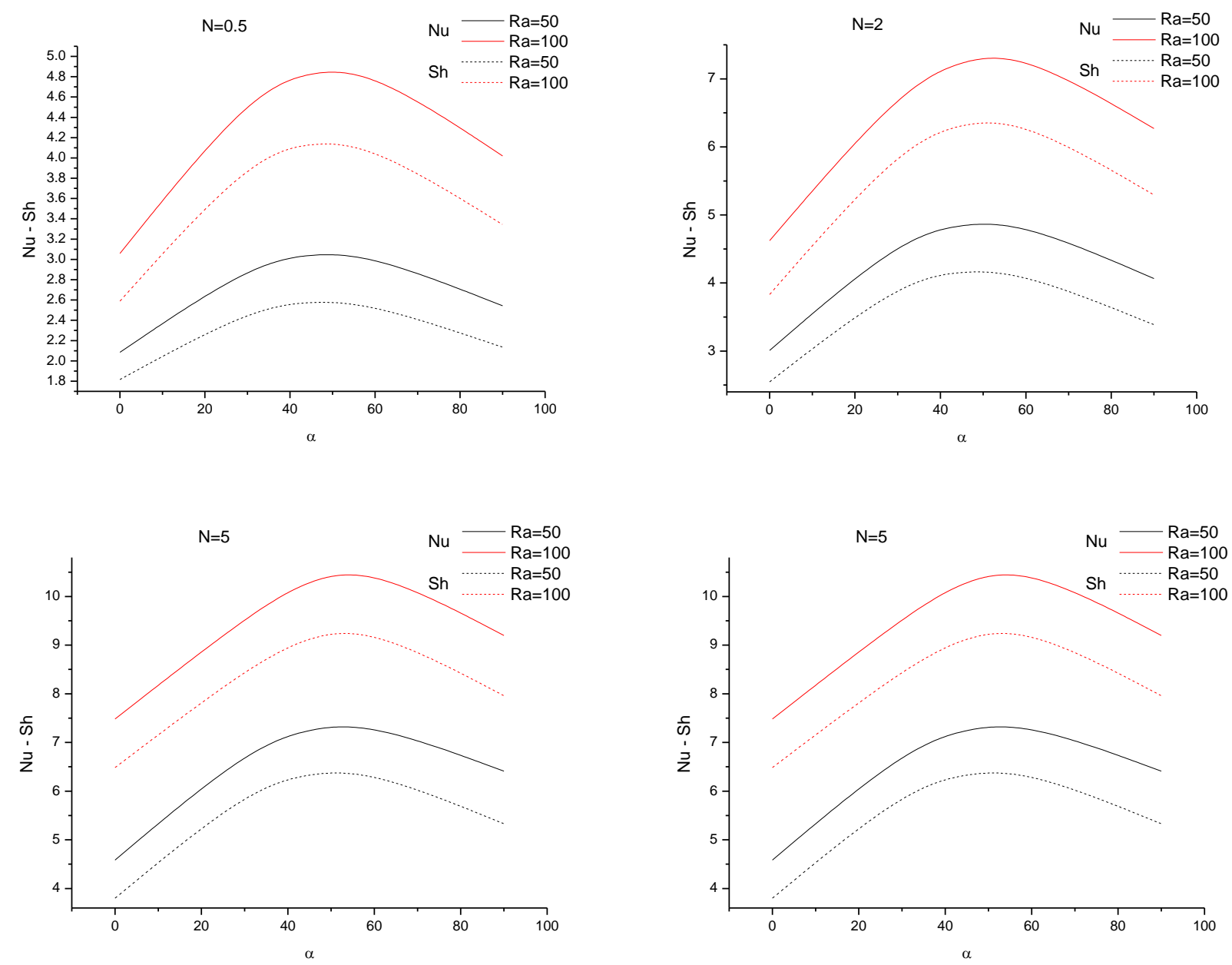

Fig. 5: Global Nusselt Number Versus Inclination Angle $\alpha$ and for $R a=50$ and 100 (A) $N=0.5,(B) N=2,(C) N=5$. 


\section{Conclusion}

In terms of flow structure, temperature levels and concentration levels, strong changes occur in the square enclosure when changes are made on the Darcy-modified Rayleigh number and on the inclination angle of the enclosure. Increasing the source term of the vertical momentum equation, by increasing the Darcy-modified Rayleigh number or by increasing the buoyancy ratio, always leads to increases on the heat and mass transfer performances of the enclosure. Very different behaviors are obtained for the combined global heat and mass flows that cross the enclosure. In what concerns the heat and mass transfer performances of the square enclosure, some main aspects should be mentioned. Selected combinations of the buoyancy ratio and inclination angle can lead to considerably high heat and mass flows through the enclosure, and some combinations of these parameters can even lead to the maximum allowable heat and mass transfer. It is thus present a maximum transfer performance, which is of crucial importance when the enclosure is to be used as a transfer promoter. However, other selected inclination angles from the foregoing ones, can lead to essentially unchanged poor transfer performances of the enclosure.

\section{References}

[1] D. Nield, and A. Bejan, Convection in Porous Media, 2nd ed. Spinger-Verlang, New York Inc. 1999. http://dx.doi.org/10.1007/978-1-47573033-3.

[2] D. B. Ingham, and I. pop, Transport phenomena in porous media, 2nd ed. Pergamon, Amsterdam, 2002.

[3] V. Trevisan, A. Bejan, Mass and heat transfer by high Rayleigh number convection in a porous medium heated from below, Int. J. Heat Mass Transfer, vol. 30, 2341-2356. 1987. http://dx.doi.org/10.1016/0017-9310 (87)90226-2.

[4] M. Mamou, P. Vasseur, Thermosolutal bifurcation phenomena in porous enclosures subject to vertical temperature and concentration gradients, J. Fluid Mech. 395, 61-87. 1999. http://dx.doi.org/10.1017/S0022112099005753.

[5] M. Bourich, M. Hasnaoui, A. Amahmid, Double-diffusive natural convection in a porous enclosure partially heated from below and differentially salted, Int. J. Heat Fluid Flow 25, 1034-1046. 2004. http://dx.doi.org/10.1016/j.ijheatfluidflow.2004.01.003.

[6] M. Bourich, A. Amahmid, M. Hasnaoui, Double diffusive convection in a porous enclosure submitted to cross gradients of temperature and concentration, Energy Conversion and Management 45, 1655-1670. 2004. http://dx.doi.org/10.1016/j.enconman.2003.10.003.

[7] AA. Mohamad, R. Bennacer Natural convection in a confined saturated porous medium with horizontal temperature and vertical solutal gradients. Int J Thermal Sci 40:82-93, 2001. http://dx.doi.org/10.1016/S1290-0729 (00)01182-0.

[8] Mahidjiba, M. Mamou, P. Vasseur, Onset of double-diffusive convection in a rectangular porous cavity subject to mixed boundary conditions, Int. J. Heat Mass Transfer, vol. 43, pp. 1505-1522, 2000. http://dx.doi.org/10.1016/S0017-9310 (99)00238-0.

[9] L. Kalla, P. Vasseur, R. Benacer, H. Beji, R. Duval, Double diffusive convection within a horizontal porous layer salted from the bottom and heated horizontally. Int. Comm. Heat MassTransfer 28, 1-10. 2001. http://dx.doi.org/10.1016/S0735-1933 (01)00207-X.

[10] V.A.F. Costa, Double-diffusive natural convection in parallelogrammic enclosures filled with fluid-saturated porous media, Int. J. Heat Mass Transfer, vol. 47, pp. 2699-2714, 2004. http://dx.doi.org/10.1016/j.ijheatmasstransfer.2003.11.031.

[11] Patankar S., Numerical Heat Transfer and Fluid flow, Hemisphere, New York, 1980. 